\title{
Automatic Detection of Body Landmarks in Human Body Scans Lower Limb Analysis for Biomedical and Footwear Applications
}

\author{
Alessandra TURSI, Gregorij KURILLO, Ruzena BAJCSY \\ University of Berkeley, Berkeley CA, USA
}

DOI: $10.15221 / 17.179$ http://dx.doi.org/10.15221/17.179

\begin{abstract}
This paper presents a pilot study of detection and deformation tracking of 3D body landmarks. In particular, the primary goal of this research was to develop methodology for automated design of individualized 3D printed ankle and foot orthoses for persons affected by Duchenne and Becker muscular dystrophies. The results of this study could have further implications on the design of not only the static orthoses but also active exoskeleton systems and general body deformation tracking.

Although body scanners are becoming increasingly popular in the recent years, due to new advances in 3D sensing, there are several outstanding issues related to the automated extraction and standardization of body measurements. Modern body scanners are able to virtually reproduce body shapes with an extreme accuracy. However, the result of a body scan is an indistinct 3D mesh, usually without any easily recognizable body landmarks that could provide consistent and robust measurements of the anthropometric parameters related to the particular body part. Although some of the body scanning software packages provide automatic or semi-automatic segmentation and extraction of different body measures, the algorithms are proprietary and do not easily compare between different systems.

In summary, the important open questions are the following: 1) how to uniquely recognize the same points on scans from different people in order to extract comparable measurements, 2) how to extract the same measures starting from two body scans of the same person, acquired and processed by different body scanning hardware. This paper first briefly describes the state of art in body scanning and body measurement extraction while focusing in particular on lower limbs. The lower limbs are one of the more challenging body parts for scanning as there are very few easily recognizable body landmarks. Majority of the landmarks described in the literature require palpation to locate them and therefore cannot be detected purely from visual data. Presented analysis is focused on first obtaining $3 \mathrm{D}$ scans of the foot, ankle and lower leg in different configurations (e.g. flexion/extension) using a custom-developed contraption that allows for scanning in a secure static position. In our tests, we used the most common landmarks reported in medical and design literature. The scans obtained in the different configurations are then aligned based on the landmarks while the algorithm extracts the skin surface deformation in different cross-sectional plains. Based on the change in curvature, it is possible to extrapolate this information across the surface of the lower limb to estimate how much soft tissue deformation is exhibited during the observed configuration change.

By quantifying the deformation across the surface of the lower limb, it is possible to determine the sections of the limb where the deformations are small. This information is crucial for customized design of biomedical and footwear products. For example, the areas that exhibit low deformation/movement can be put in close and more rigid contact with the orthosis, while the areas with large deformation require additional padding and softer materials to prevent skin irritation and discomfort.
\end{abstract}

Keywords: Bodyscanner, body landmarks, body tracking, laser scanner, parametric analysis

\section{Introduction - The objective of the research}

Human body scanner technology is relatively new, but its fast growth indicates increased interest in the topic with a wide range of possible applications. Modern instruments are becoming more and more accurate, however, the process and reliability of the extracted body measures lacks transparency. Body scanner vendors typically provide proprietary software designed for a particular scanner, preventing the users from comparing results of the same body measurement obtained from different devices. This research is motivated by the aforementioned issue while aiming to develop a general methodology for automatic extraction of body measurements and tracking of deformations that can be compared across scans, devices, and persons. Although existing software packages provide tools to compare scans, they typically require scans to be obtained in the same body configuration. One of the outcomes of this research is to generate a visualization approach that can be used to compare scans in two different body configurations, in order to visualize the deformations 
across the body scan. Although our focus during the development of this algorithm was on the lower extremities, we demonstrate that the same method can be applied to other parts of the body, such as the upper extremities. The proposed algorithm and results presented in this paper were developed using Rhinoceros software and its plugin for parametric design Grasshopper; however, the same logic can be implemented with other tools or programing languages.

\section{Related works}

\subsection{New challenges for 3D body scans}

Despite only recent introduction to the market, human body scanners are being used in an incredibly wide range of professional and consumer applications, including in healthcare (e.g., non-invasive diagnostics, customized orthotics and prosthetics, design of implantable products, etc.) and fitness (e.g., tracking diet regimes, apparel and footwear fitting and customization). Other areas include anthropology, entertainment, Virtual and Augmented Reality. There are several challenges for using body scanners in these applications, including the need for higher accuracy, handling of occluded areas during scans, such as armpits and crotch, that are difficult to acquire in a common position, acquisition of dark colored surfaces, such as eyes and hair, and robustness to small movements during prolonged scanning. ${ }^{i}$ The result of the body scanning, regardless of the brand or technology used, is an indistinct point cloud or 3D mesh that represents a collection of coordinates with no semantic interpretation. This represents the greatest challenge as the interpretation, segmentation, and tracking depend by and large on the application context.

Body scanner segmentation - Defining a standard and uniform way for body segmentation can be a powerful tool for further identification of body landmarks. The main requirements for body scan segmentation are:

- $\quad$ being robust to different body types, gender, race, age, weight, health conditions;

- being able to perform segmentation in at least the most common human body configurations, such as standing, lying down, sitting, as well as with predictable little variations in the posture;

- being able to solve for missing data, such as small holes and cone shadows, considering that for having a full body scan, the human has to be acquired in more than one positionii.

One of the first and more robust studies on human body segmentation was developed by Nurre The author explains how to perform 3D human model segmentation into six parts: head, two arms, two legs and torso, using evaluation of the topology of horizontal sections and curvature moment analysis. The approach however requires the user to be positioned in a specific body pose. A more robust method that works for various body configurations was presented by Xiao ${ }^{v, v i}$ et al. and Wergh N. vii

Body Landmark Identification - The main problem in the identification of body landmarks is that many of these points are not immediately identifiable visually. Many of the anthropometric landmarks are often based on skeletal features, which can only be identified via touch or compression of the skin, or are based on geometrical formulas for their indirect approximated localization (e.g. based on height and weight scaling).

To date, the most advanced results concerning the recognition of points of reference have been developed for the face, while no general approaches have been developed for other parts of the body. One of the largest general body landmark identification projects was CAESAR projectivii, where 73 anthropometric locations were located from body scans in a group of 5000 people. An important study on different approaches for body landmark identification is described in detail by Dekkerix, who used the identified landmarks for high-level body segmentation. The author summarizes the body landmarks identification into three approaches:

- Template matching. This method can be applied when the shape of the scan has relatively small variability in comparison to the given model (template). Point Distribution Models can be used to adapt the template to single bodies. The parametric template is aligned with the real points of the scan, consequently defining the position of the landmarks. ${ }^{\times}$This approach works only in a limited range of variation, while requiring the scan to be obtained in the same position. Further adjustments are also needed for each body type.

- Curvature calculation, or feature discriminant functions. This method is performed locally on 3D mesh, by finding certain features, such as convexity, points of discontinuity, Gaussian distribution, or curve characterization, using Bezier curves that, after fitting on the body shape, indicate the relative location of the landmark as the peaks of the curves. Its main drawback is the complexity of the system. ${ }^{\mathrm{xi}}$ 
- Function fitting. In this method, the 3D mesh is transformed into a system of body splines that generate a tessellation on the surface while simplifying the computations of the areas and volumes. Next, the body is cut in sections horizontally and vertically and the curvature of each section is used to determine the location of body landmarks. This approach is particularly useful in healthcare environment as it provides a rapid and reliable analysis.

Other research that relies on the evaluation on body sections are the ones of Lars and Caixi and of Zhong and $\mathrm{Xu}^{\mathrm{xiii}}$, but they both focus only on a couple of body landmarks each.

In conclusion, an interesting study that uses a combination of all the techniques previously indicated and that shows clearly how extended the range of application of these technologies is, was developed by Law J. xiv. It uses robust body segmentation to drastically reduce the accuracy of some intimate body parts and blur them to assure privacy to the users. The method relies on modern concept of function fitting, curvature calculation and template matching in conjunction with ancient Vitruvian theories of "Intrinsic Proportions".

\subsection{Extraction of reliable measures}

Most of the body scanners available on the market are equipped with software that provides the extraction of selective body measurements, but very few, if not none of them, provide detailed and undisclosed information concerning how these measurements are calculated and how the starting landmarks are located. Without this information, it is impossible to obtain comparable measurements with two body scans of the same person acquired with two different machines.

The research on this topic started from the study of anatomical landmarks on lower limbs and a schedule survey on the most common lower limb measures in medical and commercial applications. Considering the anatomical landmarks on the foot, among the several foot measurements definitions, we relied on the 22 anatomical points defined by the INFOOT research ${ }^{x v}$. These points have a specific and unique correspondence with clear anatomical features of the foot and therefore they can be easily replicated.
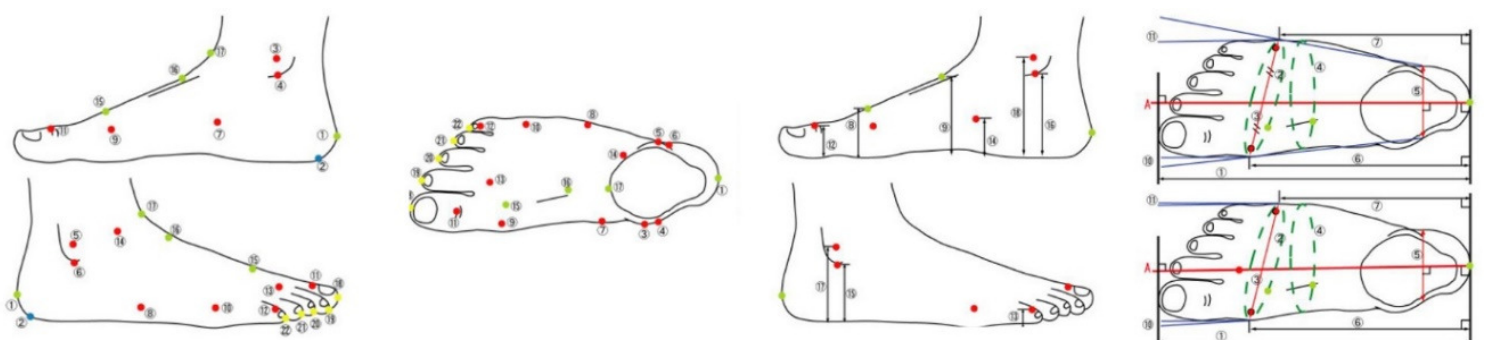

Fig. 1. Measurement definition for INFOOT_I-Ware Laboratory.

For garment design, the standard measurements usually refer to the ISO 8559 standards ${ }^{x v i}$. Since the objective of the research is the study of orthoses for people affected by Duchenne Muscular Dystrophy, we focused in particular on the measurements required to produce AFO, Ankle Foot Orthoses. As you can see even in this field, each company requires different measurements to produce customized orthoses.
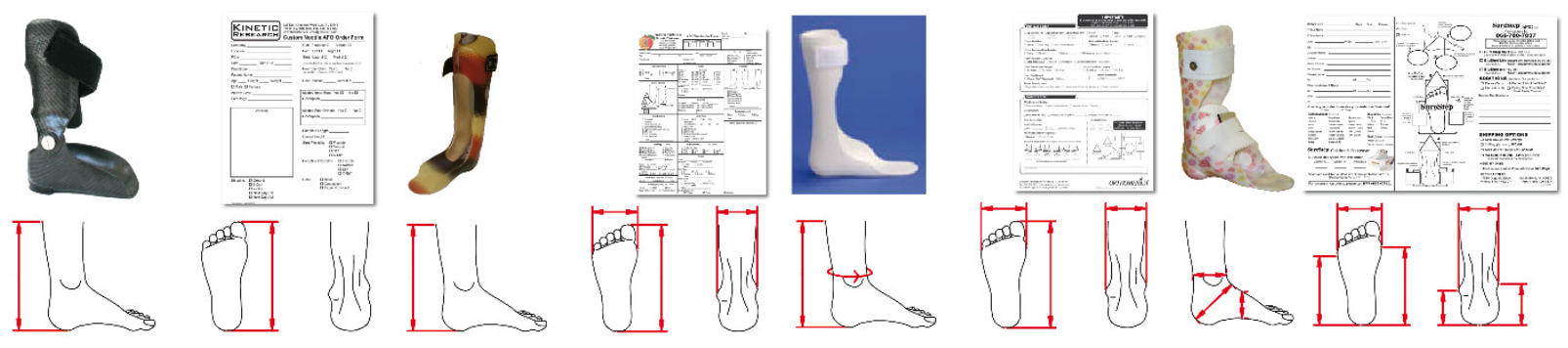

Fig. 2. Comparison of the measures required by AFOs companies to build customized orthoses.

In general, AFO manufacturers typically require the following measurements: (a) brace height (to be defined by the orthopedist), (b) foot length, (c) width at metatarsal heads, (d) posterior calcaneus to apex of 5th metatarsal head, (e) posterior calcaneus to apex of 1st metatarsal head, (f) high to apex of lateral malleolus, (g) high to apex of medial malleolus, (h) ankle diameter - smallest super malleolar circumference, (i) diagonal circumference at apex of calcaneus, and (j) midfoot circumference at apex of arch. 


\subsection{Lines of non-extension}

Although we were initially only interested in the extraction of foot measurements from body scans, it became clear that another important issue for the design of orthoses is the study of body lines of non-extension. These identify all the portions of the body area that move less when we perform movements and actions. Studied initially for the space suits design ${ }^{\text {xvii, }}$, this research proves its validity and moves a step toward taking advantage of new technologies to show the amount of movement of the soft tissue. In this sense, a research that already starts exploring the field of lines of non-extensions for lower limbs, functional at the design of Ankle Foot Orthoses, was developed by the Universidade Técnica de Lisboa ${ }^{\text {xviii. }}$

\section{Preparation of the test}

\subsection{Different scanners used}

The acquisition of lower limbs was performed with several different scanning devices as shown in Fig. 4. In this context, we will not examine in detail the different results obtained by each device and their potentials and peculiarities. The aim of this chart is to provide an overview of different technologies. In general, scanners such as Microsoft Kinect prove to work better to acquire bigger environments than a single body part but they are particularly effective in tracking movements, while devices such as iSense 3D Scanner or XYZprinting Handheld 3D Scanner performed better for our needs. Moreover, the Structured light scanner TechnoMed has good accuracy but does not provide texture information needed to locate targets on the skin. The system is only able to acquire location of special highreflectance targets. Among the structured light scanners, the Artec $3 \mathrm{~d}$ scanners have certainly better performances, with an accuracy of $0.05 \mathrm{~mm}$ or a resolution of $0.1 \mathrm{~mm}$, but their cost of about $\$ 25,000$ was prohibitive for this application.

In conclusion, we also evaluated a photogrammetric approach for 3D scanning using $20-25$ photos acquired with a Nikon digital camera and even an IPhone. Comparison of the acquired model with a model obtained by a laser scanner had a standard deviation of avg. $2 \mathrm{~mm}$. In photogrammetry, a higher attention of the environmental conditions, of the lighting and of the settings of the camera can really make a significant difference in the quality of the reconstructed model.

\subsection{Construction of the support for the foot scans}

During the scans of the lower limbs in a stretching position, we found that it was better to have a solid and standing support to avoid the natural movements of the body related to muscles' activity. The support must be as transparent as possible, in order not to occlude the body during the scan.

The main requirements of the supports are:

- Remain stable during the acquisition and support the weight of the lower limb.

- Be adjustable in all spatial directions, to proper orient the lower limb, even considering potential deformations related to various health conditions.

- Be adjustable in height, to meet different human size. The platform has to reach an adequate height the person scanning the subject, to allow him to turn all around and below the lower limb with the scanner in his/her hand.

- Be as transparent as possible, to avoid shadow cones on the skin. ${ }^{\text {xix }}$
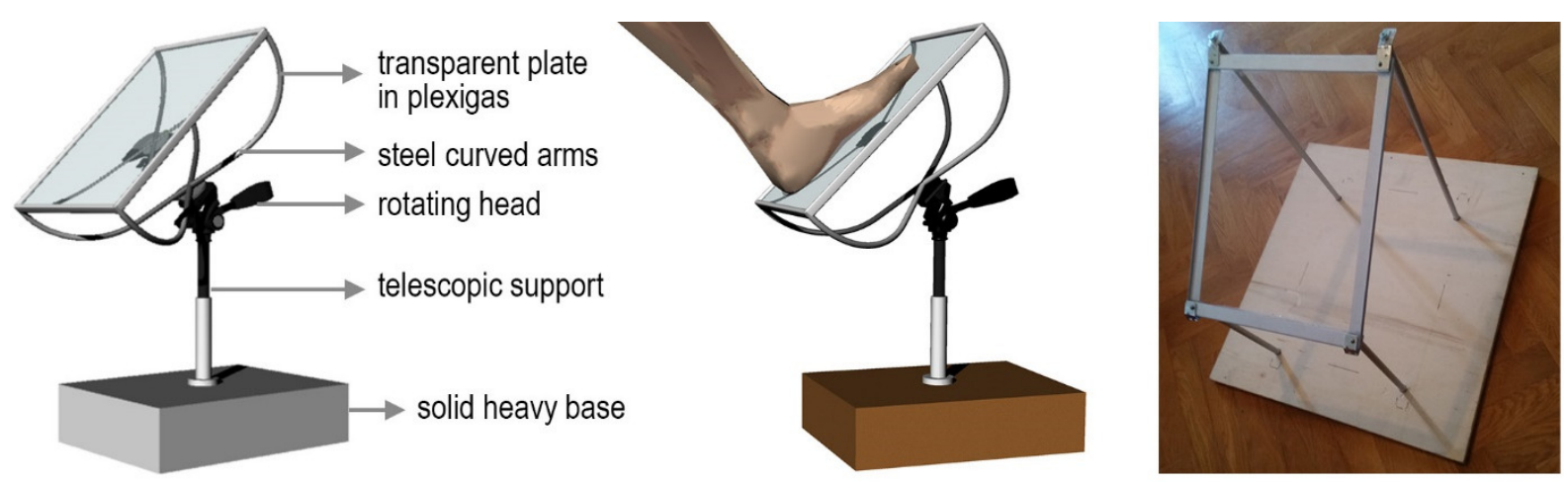

Fig. 4. Initial design concept and realization of the foot support for the laser scanner acquisition 


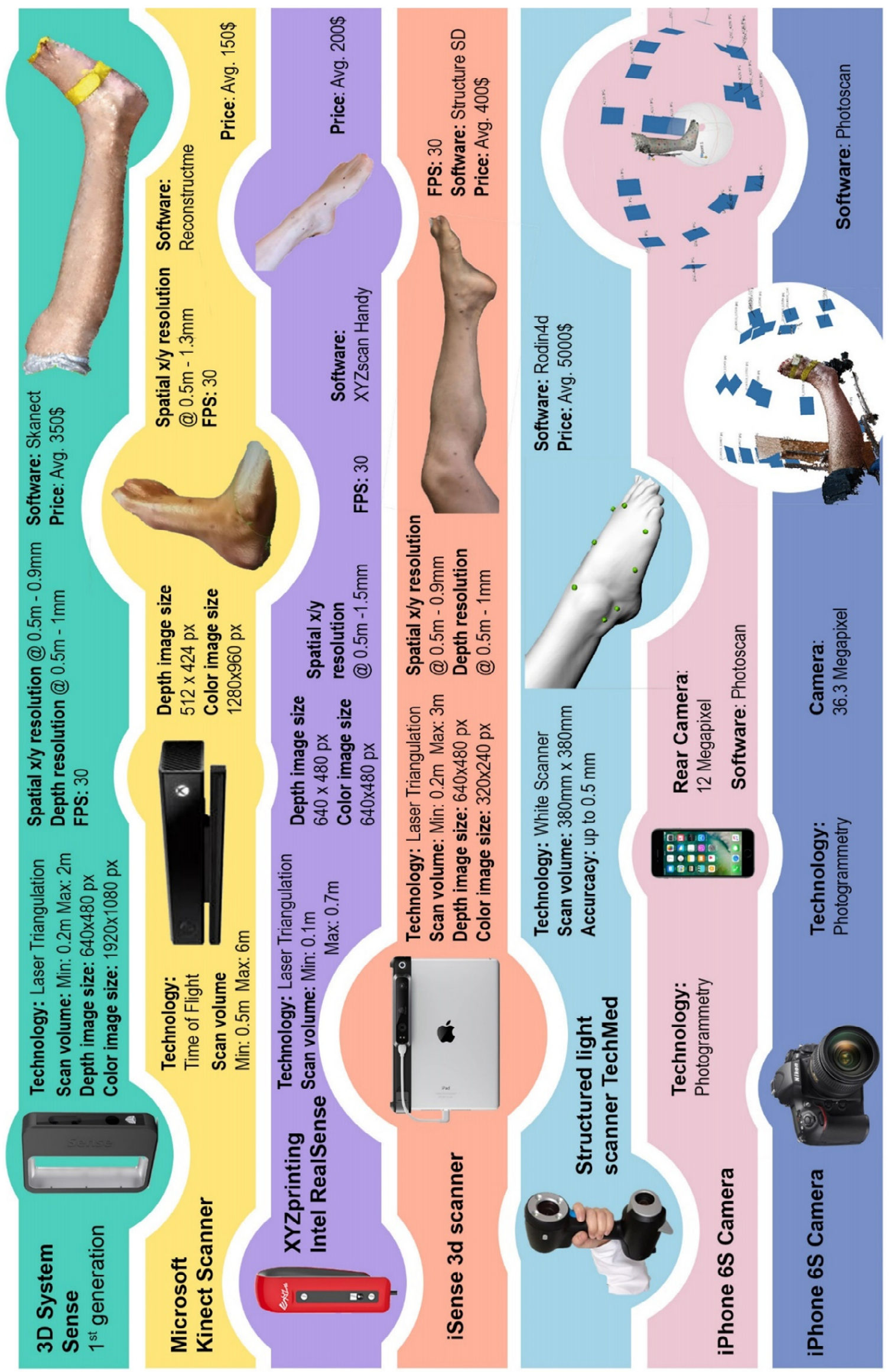

Fig. 3. Overview of all the different instruments and techniques used to acquire the geometry of the lower limb, with a synthetic comparison of the main features of each device. 
The first idea was composed by a plate made of Plexiglas, steel curved arms and a rotating head that works as the head of a tripod and it allows a perfect control of the orientation of the plate in all three spatial directions ${ }^{\mathrm{xx}}$. The telescopic support needs to extend to match the height of the person and even to facilitate the movements of the operator that has to scan the foot sole. The base can be in different materials but it should be quite heavy to counteract the push of the foot, without overturn.

To simplify the manufacturing process of the initial design, four telescopic legs and the rotating hinges attached to the plate were used to allow perfect customization with the desired position of the foot during the indirect survey acquisitions.

\subsection{Definition of lower limb landmarks and measures}

To assist with the comparison of scans in different positions, we applied markers to the skin. Different layouts and methods for landmark identification were used to optimize the algorithm for the comparison of the shapes.

In particular, the following conditions were tested (Fig. 5):

1. Automatic detection of lower limbs landmarks, mainly by curvature analysis of emerging points;

2. 22 points on the surface, as indicated by the INFOOT research, marked on the skin;

3. 22 points on the surface, detected thanks to high reflectance markers attached on the skin;

4. 22 INFOOT's points plus other 4 anthropometrical points around the knee and a regular grid all over the lower limb, until the knee, at an interval of avg. $5 \mathrm{~cm}$;

5. The same 26 anthropometrical points plus a grid of avg. $1,5 \mathrm{~cm}$ marked on the skin;

6. The last series of tests took as body landmarks: 4 or more points located at the metatarsal toe section, 4 or more points around the ankle diameter, that is the smallest super malleolar circumference, 4 or more on the diagonal circumference at apex of calcaneus, 4 or more on the midfoot circumference at apex of arch, 4 or more on the diagonal circumference at apex of the knee, 4 or more at the bottom of the knee, perpendicular to the tibia, 4 or more at the top of the knee, perpendicular to the femur.

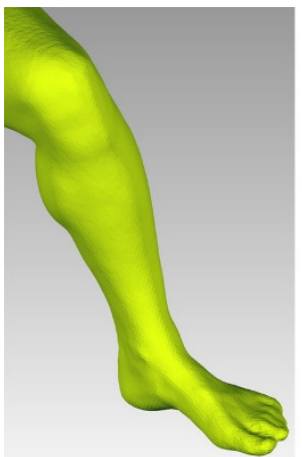

1.

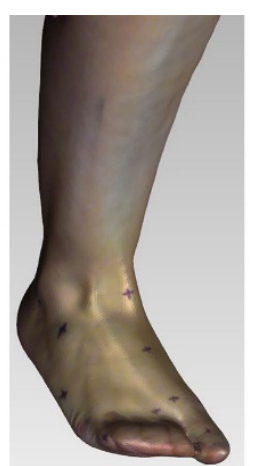

2.

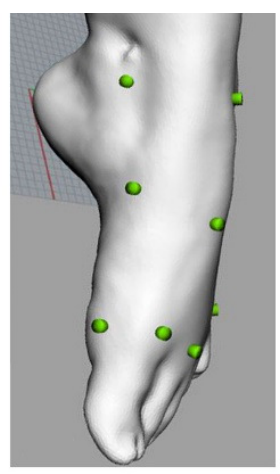

3.

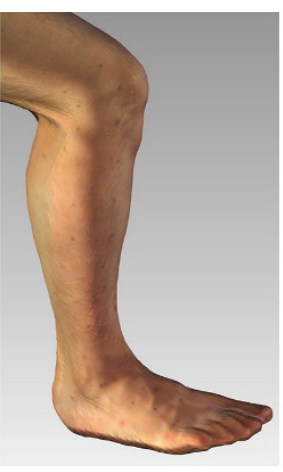

4.

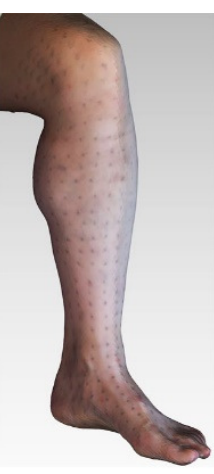

5.

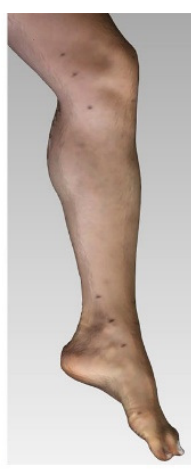

6.

Fig. 5. Overview of all the different set of body landmarks used during the tests.

It is important to note that the final algorithm that we present in this paper is independent of the method used to acquire the landmarks; however, by having physical markers directly applied onto the skin, the identification of the points is simplified.

\subsection{Acquisition of the shapes of lower limbs in different positions}

For the data acquisition, the foot was positioned in the following three positions: relaxed, plantarflexion (foot flexed downward toward the sole), and dorsiflexion (foot is in backward flexion bending, in the direction of the dorsum).

These configurations were chosen because they represent the most common three positions of the foot during a walk and they represent quite well the possible range of deformations of the soft tissue of the lower limbs.

\section{Results and discussion}

\subsection{Cleaning and adjustment of the mesh}

The body scan acquired with the ISense 3d scanner, produces several different output files (total average data size is 5- 10Mb): (a) "Preview", a JPEG file with an image of the scanned area; (b) "Model" in Wavefront OBJ file with the 3d mesh, (c) JPEG file for the texture, (d) MTL file with the 
definition of the material characteristics of the texture mesh. These files can be processed in any 3D modeling software. We used the software Geomagic Wrap 2015 to edit the perform cleaning of the data. After importing the model, the 3D model is already registered in a single mesh or point cloud, with its own reference system and texturized. The first operation is to clean the model and delete all the surveyed elements that we do not want to consider in the process. Then, we fill small holes that could be due to cone shadows, or errors in the acquisition. This semi-automatic process must be done carefully, closing only very small holes in the mesh and being sure that the new triangles continue the curvature and the shape of the boundaries without introducing any artifacts into the mesh. Finally, further optimization steps, such as removal of non-manifold edges, the alignment of the normals and the final remeshing, can be performed before further processing the scans.

\subsection{Automatic orientation of body scans}

For the automatic extraction of the measurements, the first obstacle is how to properly orient the 3D model before starting with the identification of landmarks. On this behalf, there are two possible solutions:

- Position the foot always in the same location on a measured support, having at least 3 points of the sole coincident with the reference system. In this way, we can rely on these positions to orient the lower limb in the space.

- Automatically orientation of the shape of the foot starting from a scan made wherever oriented in the space. In order to solve this problem, we generated an automatic algorithm tested with more than 50 different scans, of lower and upper limbs in different positions.

The preference between one of the two methods depends on the objective of the survey. If we are not interested in the sole of the foot, or we plan to integrate this survey with a foot scanner, the person can even stand on his feet and there is no problem at all in the identification of the spatial axis. If we are scanning lower limbs in a relaxed position with arbitrary orientation (e.g. person lying on his/her back or stomach, sitting in a chair), the algorithm provides a good solution to evaluate the axis of orientation of the model, relying only on the lower leg itself.
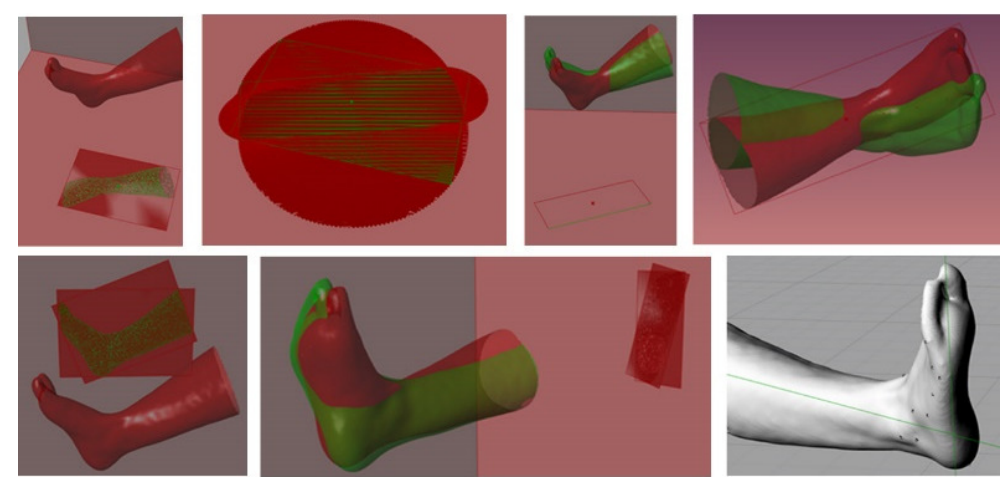

Fig. 6. Phases required by the algorithm to align a lower, upper limb or a foot in the virtual space: projection on $x$ plane and identification of the minimum bounding box, orientation along the axes of the box of the shape and repetition of the same process for the $x$ and $y$ planes.

\subsection{Recognition of landmark points}

The recognition of markers is carried out in different ways, depending on the used technique.

- Without markers, after the automatic orientation, the 3D mesh is processed for the automatic identification of the landmarks on the feet, useful for the extraction of standard measures.

- With high reflectance markers attached to the skin, the software that manages the laser scanner acquisition automatically identifies the markers and generates a layer that contains small cylinders where their centers correspond to the centers of the markers.

- With markers drawn on the skin, all the markers visible on the texture need to be associated one by one with a 3D point feature generated in the model. The downside of this method is that, if the scan was not acquired with proper light conditions and in a real static position, the generation of the mesh can have some misalignments with the actual location of the landmarks.

\subsection{Extraction of standard measures}

Other algorithms were set in order to automatically reproduce, starting from a 3D lower limb scan, with or without markers, all the most common measurements useful to describe the body. All the 
measurements were measured multiple times by hand, trying to find the best match between the average results obtained by the hand measurements and the automatic anthropometric results given by the software. It is important to notice that a relevant discrepancy between an automatic generated measurement and a hand measured one has not necessary to be considered an error, since hand measures can be difficult to reproduce always with the exact same value.

\section{Comparison of the same body in different positions}

\subsection{The final method for the colorization of the mesh}

The results presented in this paper are the latest iterations of an ongoing research aimed at comparison of the body scans captured in different configuration and the tracking of the soft tissue deformations across scans. Starting from a series of three scans of the same leg in a relaxed position, plantarflexion and dorsiflexion, the test demonstrates how it is possible to understand, visualize and measure the deformation of the soft tissue that occurs in the lower limb during such movements. The presented method relies on the localization of 3 anchor points per each joint, coincident with bone landmarks and to additional points before and after the joint useful to define the course of normal planes at the joint and help the algorithm in the description of the changes in shape of these areas. In particular 7 cutting planes were used to segment the mesh of the lower leg. Each cutting lane is defined by at least four points, in order to have an extra point for verification. These points can be:

- automatically identified on the skin, thanks to an evaluation of the curvature of the surface,

- manually marked on the skin before proceeding to the scan, applying high reflectance markers that can be automatically recognized by the software

drawn directly onto the skin and manually recognizing in a post processing phase.

In the reminder of this section, we describe the general steps for the segmentation and deformation tracking of the aforementioned body scan data and given surface key points (Fig. 7). The algorithm is designed to work without any manual input, without any constraints with regard to subject's limb size, age, health condition, or skin colors. The only requirement is to avoid "extreme" body configuration, e.g. full knee flexion.

Phase 1: Acquisition of the shape, preparation of the mesh and identification of the landmarks For this test, the markers were drawn directly on the skin and no support was used to lay the lower limb during the seconds of acquisition. After the localization of the landmarks, the ISense 3D scanner was used to acquire the three positions of the leg. Therefore, the resulting textured 3D meshes were processed, cleaned, and optimized for the analysis with the parametric design algorithm.

Phase 2: Association of the geometries and definition of the landmarks and cutting planes After the import of the geometry in the software Rhinoceros and the launch of its plugin Grasshopper, the external data of the mesh and the points are associated to the algorithm. This is the only operation requiring manual input. Once all the external data are associated, the algorithm automatically calculates and visualizes the deformations. Each group of minimum 4 points along the section needs to be associated separately to generate the average cutting plane between them.

Phase 3 - Identification and segmentation of the "skeleton line"

The fillet of the centers of each drawn section composes the middle line, the hypothetical "skeleton" of the lower limb. This nurb is divided by the number of proposed horizontal planes of the tessellation while each point is associated with its portion delimited by the cutting planes.

Phase 4 - Generation of the grid of points, tessellation of the mesh

A series of planes, with the z-axis aligned along the "skeleton line", and the x-axis facing towards the first aligned picked points on the surface, is disposed along the skeleton line.

From here, a series of rays is projected from the center of each plane. They are aligned towards the surface and generating a point of intersection with the mesh.

Phase 5-Repetition of the same process for the other scans and generation of the grid of points The same algorithm is then applied to the scans obtained in non-reference configurations. Applying the same tessellation to all the scans allows for easy comparison at the level of each patch. Denser tessellation will in general produce a more accurate result. However, if the tessellation patches are smaller than the actual mesh resolution, the results will not be valid. Then, all the points of each model are collected to generate an ordinate grid of points in $x$ and $y$ directions.

Phase 6 - Calculation of the areas and comparison of the relative tassels

In the last phase, we calculate the area of each tassel generated by the intersection of the grid points with the mesh. The relative change of the area is colored as shown in Fig. 8. The red areas correspond to the portions of the skin that stretched during the movement, the yellow parts correspond to the areas with small changes, and the green indicates skin contraction and reduction of the surface. Based on this notation, we can easily identify the lines of non-extension colored with yellow. 
Acquisition of the shape, preparation of the mesh and identification of the landmarks
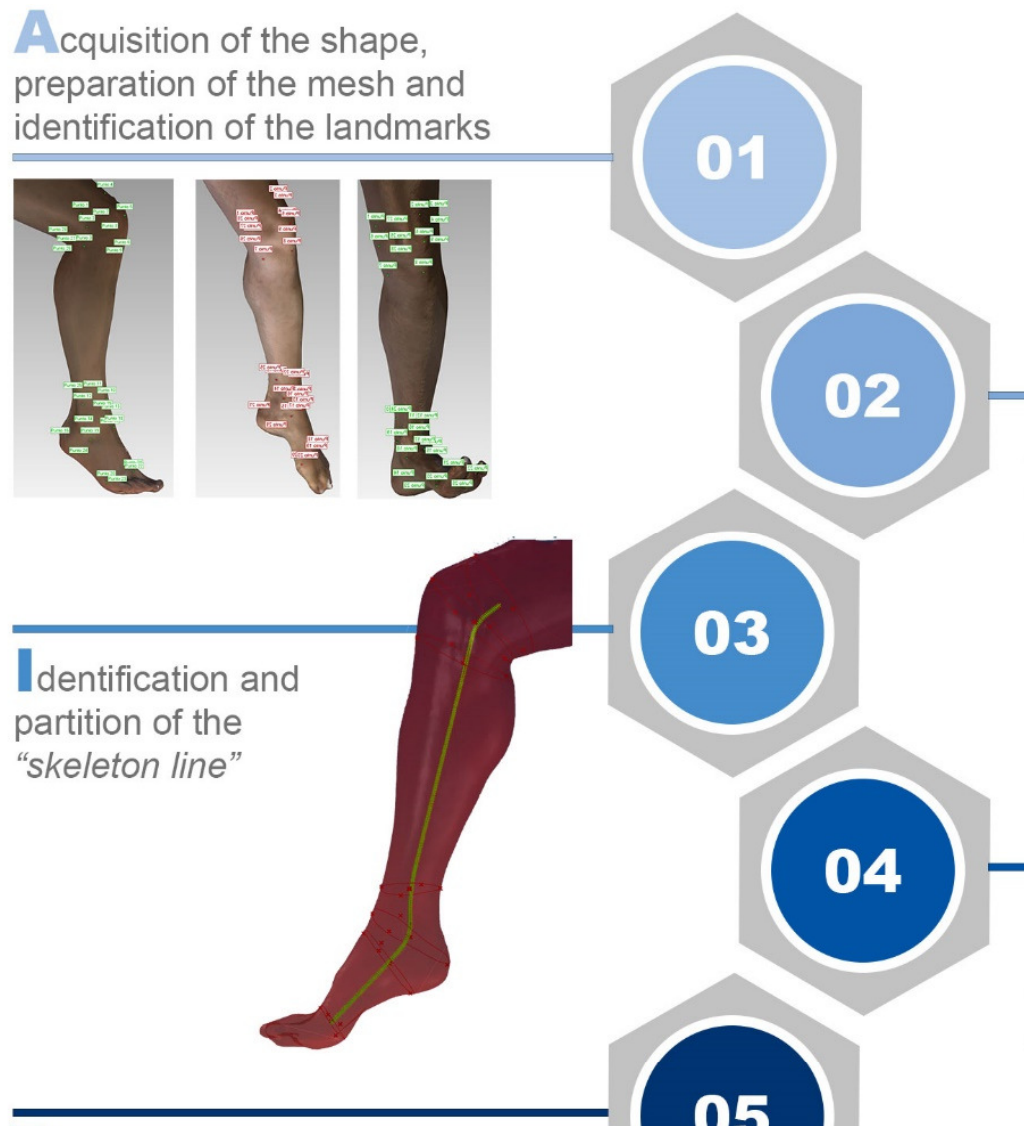

Repetition of the same process

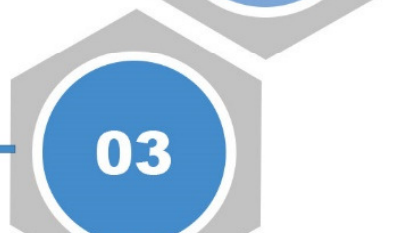

Association of the geometries and definition of the landmarks and cutting planes

for the other scans and generation of the grid of points
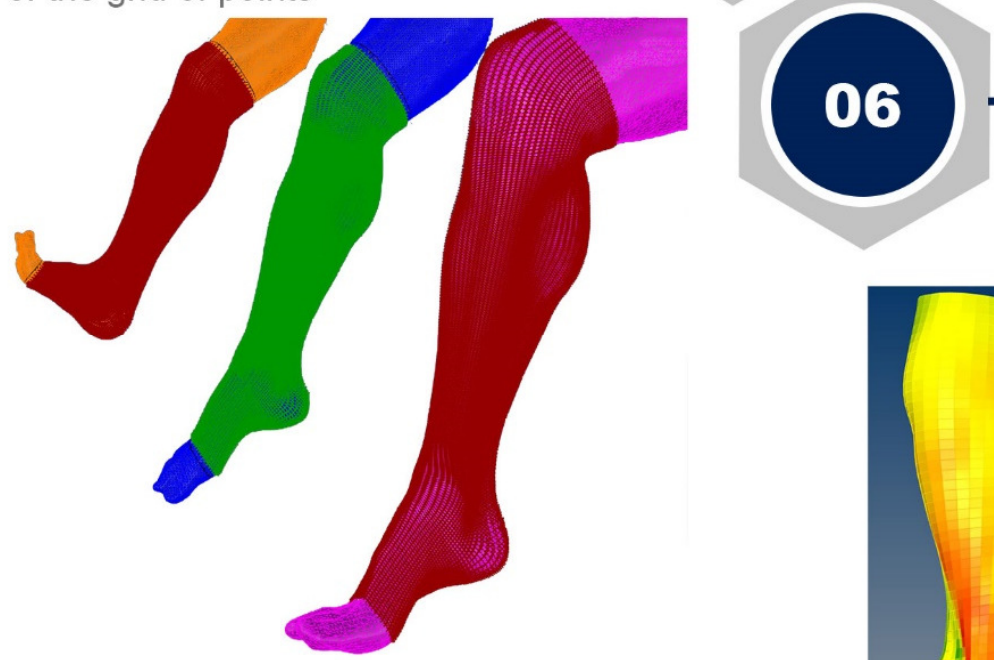

Calculation of the areas and comparison of the relative tassels. Generation of the texture.
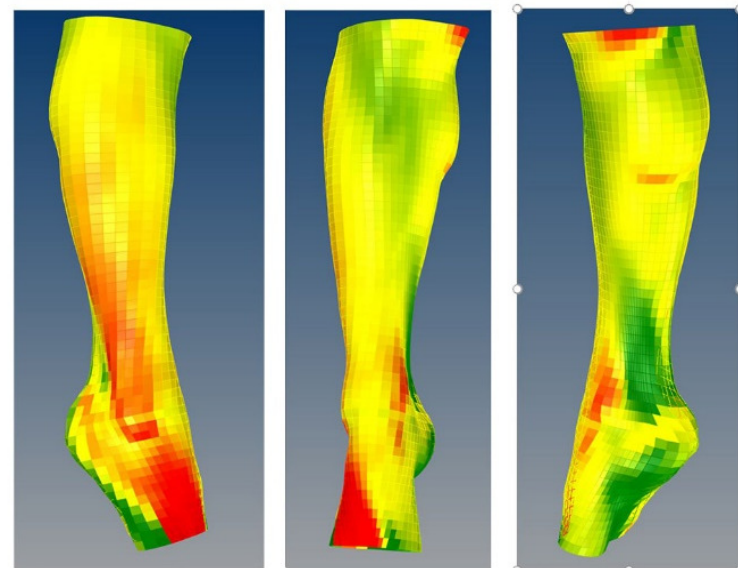

Fig. $7-8$. Schematization of the process required to elaborate the texture on a lower limb in analysis starting from one of reference. On the other page, a general view of the entire algorithm and the result on a leg in plantarflexion. The red and green areas refer to the area of extension or compression compared to the scan of the foot in a position of relax. 
Proceedings of 3DBODY.TECH 2017

8th International Conference and Exhibition on 3D Body Scanning and Processing Technologies, Montreal, Canada, 11-12 Oct. 2017

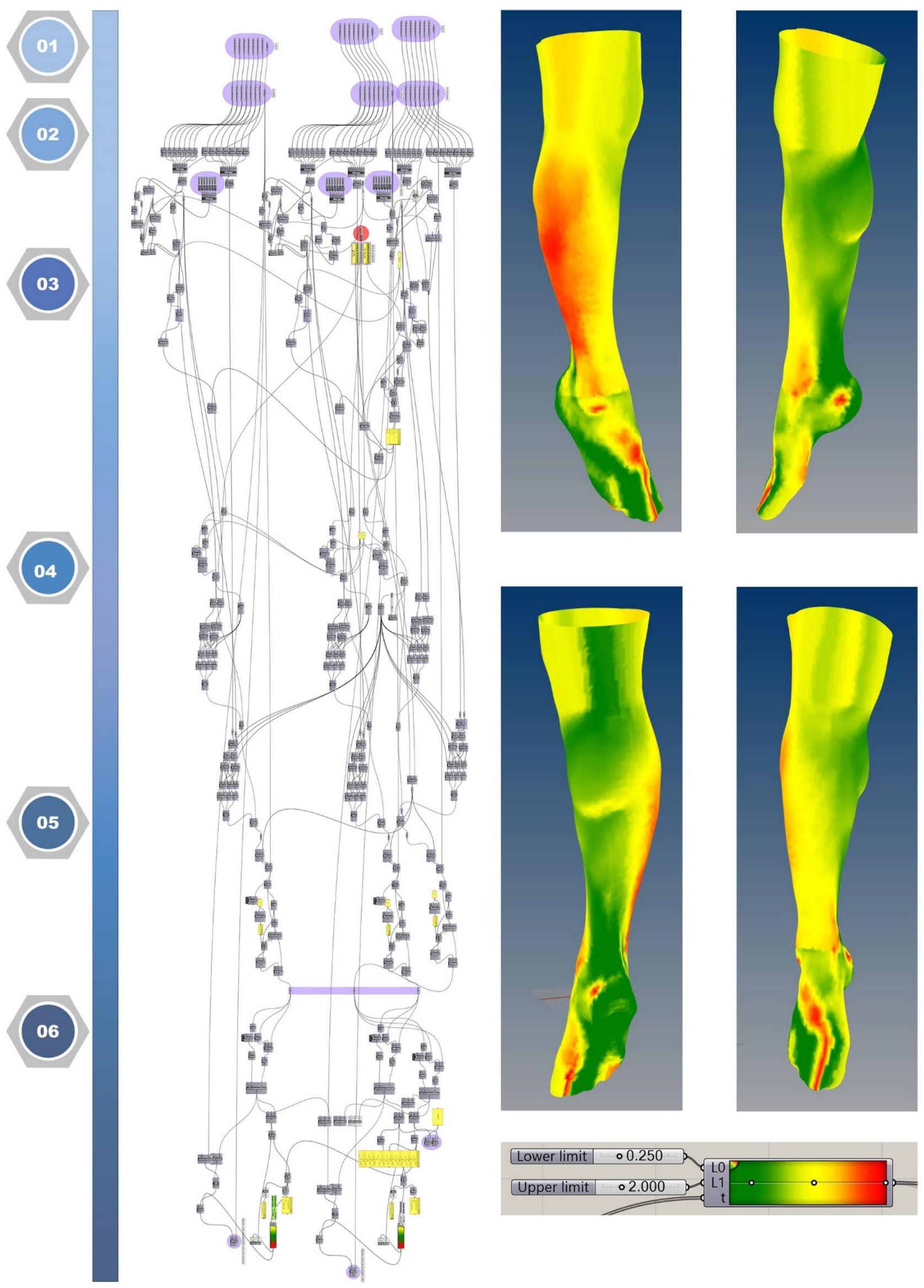




\subsection{Validation of the method with a known geometry}

To validate the method several tests were run on a synthetic model with known geometry, before and after applied deformation. For clarity, we demonstrate the results on a simple object shaped as a cylinder. The cylinder was divided into three areas. For the deformed scenario, the central area was enlarged in its medial diameter, while the diameters in the middle of the other two portions were reduced as shown in Fig. 9. We applied the same algorithm as presented earlier to the mesh and identified key points. As expected, the green areas correspond to a reduction and red areas to an enlargement in surface. Moreover, it is possible to check the validity in the colorization, manually measuring outside from the algorithm the deformation of corresponding tassels, from the original cylinder of reference to the deformed one.
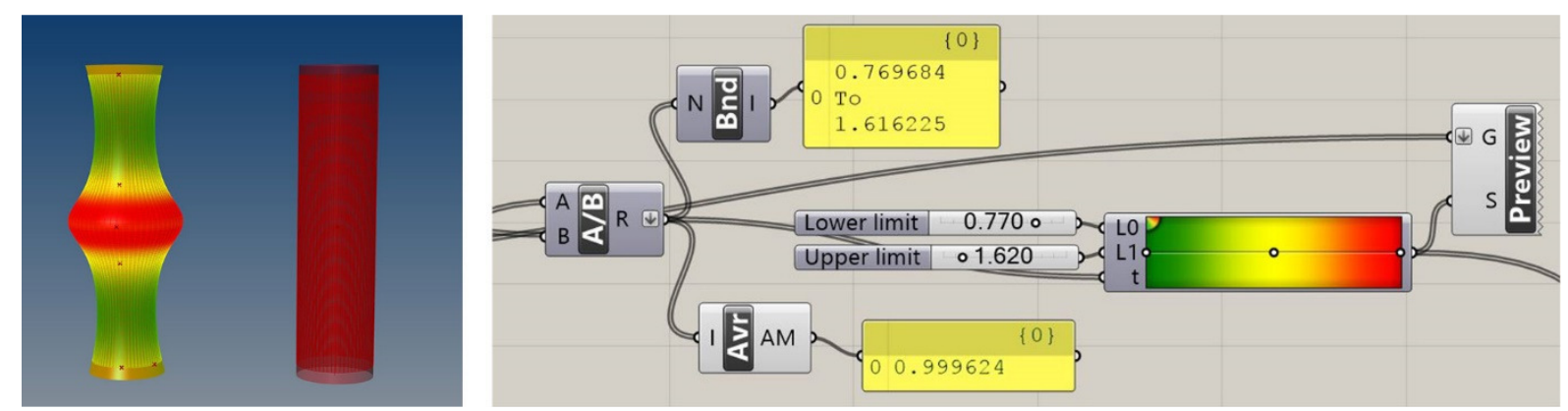

Fig. 9. Validation of the method applying the same algorithm on a known and measurable geometry.

\subsection{Application of the same method for upper limbs}

The algorithm developed for the lower limbs was, without any changes, applied also to the upper limb. The results in Fig. 10 show the deformation of soft tissue in the bending of the arm for three different configurations: relaxed position, partially bended, and 90-degree bended arm. The results show that the elbow flexion exhibits stretching of the skin in the outer elbow area and forearm (red color) and contraction in the inner elbow area (green color).

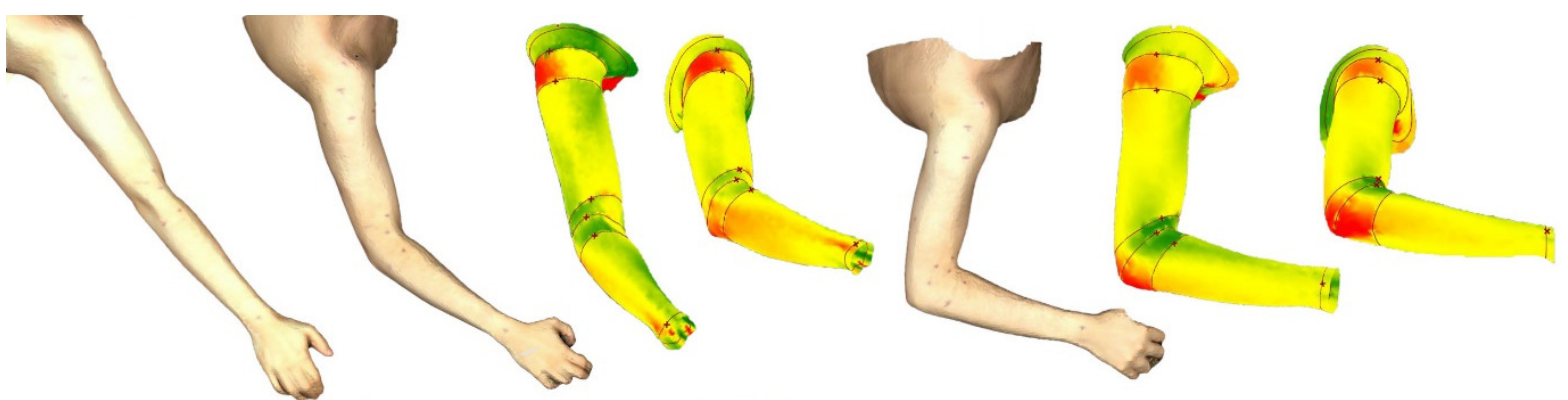

Fig. 10. Application of the same algorithm for the analysis of the upper limbs.

Red and green areas indicate the parts of stretching and compression of the skin during the bending of the arm.

\section{Conclusions - First results and future developments and applications of the research}

Presented method for the analysis of skin deformation proves to be very fast and requires no manual input after the initial association of the mesh with the algorithm. This is a crucial element in the planning of its future application on a larger scale. Thanks to the developed algorithm of the soft tissue movements in different body positions, the study of the lines of non-extension supported by modern technologies and the subsequent analysis can give a precious contribution to the soft tissue modeling. Application of soft tissue modeling theories are now broadly used in healthcare environment, for surgery planning, to manufacture orthoses, prostheses and exoskeletons, in Virtual Reality and computer animation, in footwear and clothing design, to better describe and reproduce movements.

However, our research is still in early stages and requires further tests on a larger number of healthy subjects and subjects with various lower-limb impairments to demonstrate its value in the design process of orthoses and other assistive devices. Nevertheless, the first results are quite promising and worth to be further developed. 


\section{Acknowledgements}

This Postdoctoral research was sponsored by the Italian Parent Project Onlus, for children affected by Duchenne and Becker Muscular Dystrophy. None of this work would ever been possible without their precious support. The automatic identification of lower limbs landmarks can really be the first step for the automatic and customized design of 3D printed Ankle Foot Orthoses that the people affected by this disease wear for their entire life. Thanks are due to Federico Uccelli, David Anton Saez and all the others that volunteered as subjects of the body scans. A special thanks to Alessandro Innocenti, precious guide and supervisor for the use of the Grasshopper software. And last but not least thank you to my professor Ruzena Bajicsy, to Gregorij Kurillo and Robert Matthew of the Department of Electrical Engineering and Computer Science and of Berkley, for having guided and supported this research.

\section{References}

i,ii Werghi, N., "Segmentation and Modeling of Full Human Body Shape From 3-D Scan Data: A Survey. SYSTEMS, MAN, AND CYBERNETICS - PART C: APPLICATIONS AND REVIEWS, vol. 37(n. 6). 2007

iNurre J. H., C. J. "On segmenting the three-dimensional scan data of human body", IEEE Trans. Med. Imag., vol. 18, no. 8, p. $787-797,2000$

iii Nurre, J. H. "Locating landmarks on human body scan data." Proc. Int. Conf. Recent Adv. 3-D Digit. ImagingModel, p. 289295. Ottawa, ON, Canada, 1997

iii Xiao Y., W. N. "Topological segmentation of discrete human body shapes in various postures based on geodesic distance", Proc. Int. Conf. Pattern Recognit., (p. pp. 131-135). Cambridge, U.K., 2004

iii Xiao Y., W. N. "A topological approach for segmenting human body shape", Proc. Int. Conf. Image Anal. Process., p. pp. 82-87. Mantova, Italy, 2003

iii Werghui N., Xiao Y., "Posture Recognition and Segmentation from 3D Human Body Scans", Proceedings of the First International Symposium on 3D Data Processing Visualization and Transmission (3DPVT02), IEEE, 2002

iii Robinette, K.M., Blackwell, S., Daanen, H.A.M., Fleming, S., Boehmer, M., Brill, T., Hoeferlin, D., Burnsides, D.. "Civilian American and European Surface Anthropometry Resource (CAESAR), Final Report", Volume I: Summary, AFRL-HE-WPTR-2002-0169, United States Air Force Research Laboratory, Human Effectiveness Directorate, Crew System Interface Division, 2255 H Street, Wright-Patterson AFB OH 45433-7022 and SAE International, 400 Commonwealth

Dr., Warrendale, PA 15096, 2002

iii Dekker L., D. I. "Building symbolic information for 3-D human body modeling from range data", Proc. Int. Conf. Recent Adv. 3-D Digit. Imaging Model, (p. 388-389). Ottawa, 1999

iii Suikerbuik C. A. M., T. J. "Automatic feature detection in 3-D human body scans", Digit. Human Model. Conf, (p. Paper 04DHM-52). Detroit, MI, 2004

iii Dekker L., D. I. "Building symbolic information for 3-D human body modeling from range data", Proc. Int. Conf. Recent Adv. 3-D Digit. Imaging Model, (p. 388-389). Ottawa, 1999

iiiiii J. Laws and Y. Cay "A privacy algorithm for 3-D human body", Carnegiie Mellon Cylab, Pittsburgh, PA, Tech. Rep. 06-00, 2006

iii Y. Zhong and B. Xu, "Automatic segmenting and measurement on scanned human body", Int. J. Clothing Sci. Technol., vol 18, no.1, pp. 19-30, 2006

iii Laws J., C. Y., "A privacy algorithm for 3-D human body", (Vol. Tech. Rep. 06-001). Pittsburgh, PA,: Carnegie Mellon Cylab, 2006

iii "Foot Measurements", I - Ware Laboratory, http://www.iwl.jp/main/mark_dimension.html, May 2017

iii ISO 8559 - ICS : 61.020 Clothes, https://www.iso.org/standard/61686.html, March 2017

iii Iberall, A.S., "RAND Development Corporation Report AMRL-TR-64-118: The Use of Lines of Nonextension to Improve Mobility in Full-Pressure Suits" (PDF). Behavioral Sciences Laboratory, Aerospace Medical Research Laboratories, Aerospace Medical Division. Air Force Systems Command, Wright-Patterson Air Force Base, Ohio, 1964

iii A. R. Domingues , S. P. Marreiros, J. M. Martins, M. T. Silva and D. J. Newman, Skin stran field analysis of the human ankle joint, $4^{\circ}$ Congresso Nacional de Biomecãnica, Dominigues, Ana Rita et al., Coimbra, Portugal, 2010

iii In the definition of the requirements and in some technical solution, the author referred to her previous Italian patent, $\mathrm{N}$. 102016000088180 deposited on the 30.08.2016 "Supporto per il rilievo indiretto degli

arti inferiori", of a support for the survey of lower limbs.

iiiA. Tursi (2014), Design for people affected by Duchenne Muscular Dystrophy. Proposal for a new type of night AFO, Ankle Foot Orthosis, based on 3D indirect survey and 3D printing", PhD thesis ICAR/12 at the Unversity of Ferrara, Italy

iv Nurre, J. H. "Locating landmarks on human body scan data." Proc. Int. Conf. Recent Adv. 3-D Digit. ImagingModel, p. 289295. Ottawa, ON, Canada, 1997

vXiao Y., W. N. "Topological segmentation of discrete human body shapes in various postures based on geodesic distance", Proc. Int. Conf. Pattern Recognit., (p. pp. 131-135). Cambridge, U.K., 2004 
vi Xiao Y., W. N. "A topological approach for segmenting human body shape", Proc. Int. Conf. Image Anal. Process., p. pp. 82-87. Mantova, Italy, 2003

vii Werghui N., Xiao Y., "Posture Recognition and Segmentation from 3D Human Body Scans", Proceedings of the First International Symposium on 3D Data Processing Visualization and Transmission (3DPVT02), IEEE, 2002

viii Robinette, K.M., Blackwell, S., Daanen, H.A.M., Fleming, S., Boehmer, M., Brill, T., Hoeferlin, D., Burnsides, D.. "Civilian American and European Surface Anthropometry Resource (CAESAR), Final Report", Volume I: Summary, AFRL-HE-WPTR-2002-0169, United States Air Force Research Laboratory, Human Effectiveness Directorate, Crew System Interface Division, 2255 H Street, Wright-Patterson AFB OH 45433-7022 and SAE International, 400 Commonwealth Dr., Warrendale, PA 15096, 2002

ix Dekker L., D. I. "Building symbolic information for 3-D human body modeling from range data", Proc. Int. Conf. Recent Adv. 3-D Digit. Imaging Model, (p. 388-389). Ottawa, 1999

$\times$ Suikerbuik C. A. M., T. J. "Automatic feature detection in 3-D human body scans", Digit. Human Model. Conf, (p. Paper 04DHM-52). Detroit, MI, 2004

xi Dekker L., D. I. "Building symbolic information for 3-D human body modeling from range data", Proc. Int. Conf. Recent Adv. 3-D Digit. Imaging Model, (p. 388-389). Ottawa, 1999

xiixi J. Laws and Y. Cay "A privacy algorithm for 3-D human body", Carnegiie Mellon Cylab, Pittsburgh, PA, Tech. Rep. 0600,2006

xiii Y. Zhong and B. Xu, "Automatic segmenting and measurement on scanned human body", Int. J. Clothing Sci. Technol., vol 18, no.1, pp. 19-30, 2006

xiv Laws J., C. Y., "A privacy algorithm for 3-D human body", (Vol. Tech. Rep. 06-001). Pittsburgh, PA,: Carnegie Mellon Cylab, 2006

xv "Foot Measurements", I - Ware Laboratory, http://www.iwl.jp/main/mark_dimension.html, May 2017

xvi ISO 8559 - ICS : 61.020 Clothes, https://www.iso.org/standard/61686.html, March 2017

xvii Iberall, A.S., "RAND Development Corporation Report AMRL-TR-64-118: The Use of Lines of Nonextension to Improve Mobility in Full-Pressure Suits" (PDF). Behavioral Sciences Laboratory, Aerospace Medical Research Laboratories, Aerospace Medical Division. Air Force Systems Command, Wright-Patterson Air Force Base, Ohio, 1964

xviii A. R. Domingues, S. P. Marreiros, J. M. Martins, M. T. Silva and D. J. Newman, Skin stran field analysis of the human ankle joint, $4^{\circ}$ Congresso Nacional de Biomecãnica, Dominigues, Ana Rita et al., Coimbra, Portugal, 2010

xix In the definition of the requirements and in some technical solution, the author referred to her previous Italian patent, $\mathrm{N}$. 102016000088180 deposited on the 30.08.2016 "Supporto per il rilievo indiretto degli

arti inferiori", of a support for the survey of lower limbs.

xxA. Tursi (2014), Design for people affected by Duchenne Muscular Dystrophy. Proposal for a new type of night AFO, Ankle Foot Orthosis, based on 3D indirect survey and 3D printing", PhD thesis ICAR/12 at the Unversity of Ferrara, Italy 